\title{
NOTE CONCERNANT LE TRYPANOSOMA RODHAINI WALRAVENS
}

\author{
Par P. WALRA VENS
}

Au Congrès de médecine tropicale de St-Paul-de-Loanda, nous avons décrit sommairement un nouveau trypanosome trouvé chez le pore, et lui avons donné le nom de $T$. rodhaini.

Une centaine de formes ont été mesurées et nous donnons

$$
\overbrace{}^{2}
$$
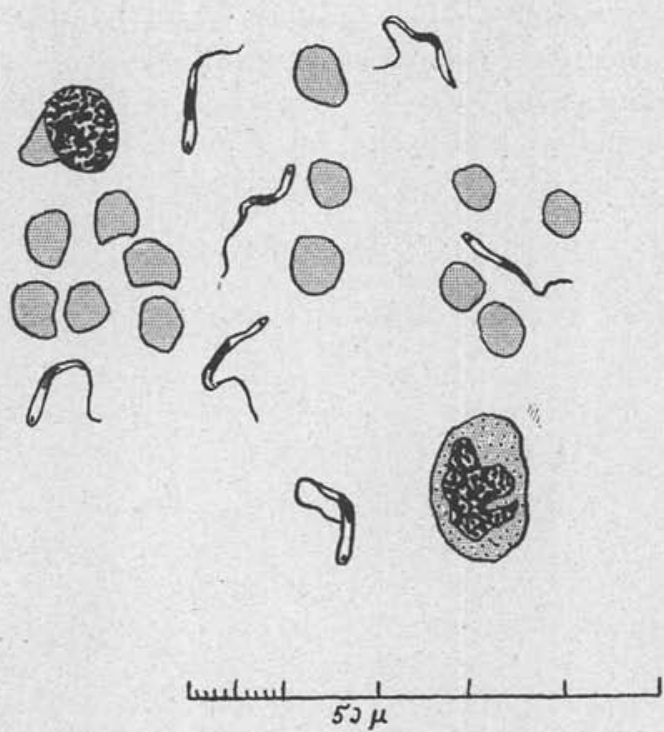

Fig. - Trypanosoma rodhaini.

ci-dessous les caractères des mensurations des diverses parties de ce trypanosome.

Flagelle: Longueur moyenne, $7 \mu, 02$; longueur maxima, $12 \mu, 5$; longueur minima, $4 \mu, 2$.

Corps : Longueur moyenne, $12 \mu, 6$; longueur maxima, $15 \mu, 5$; longueur minima, $9 \mu, 1$.

Annales de Parasitologie, T. IV, $\mathrm{N}^{\circ}$ 1. - Ier janvier 1926, p. 31-32. 
Noyau : Longueur moyenne, $2 \mu, 9$; longueur maxima, $4 \mu, 4$; longueur minima, $1 \mu, 7$; largeur moyenne, $0 \mu, 7$.

EXTRÉmité POSTÉRIEURE DU CORPS AU NOYAU: Longueur moyenne, $5 \mu, 9$; longueur maxima, $8 \mu, 5$; longueur minima, $2 \mu, 8$.

EXtrÉmité ANTÉrievre de CORPS AU NOyAU : Longueur moyenne, $3 \mu, 8$; longueur maxima, $7 \mu$; longueur minima, $2 \mu$.

Les caractères morphologiques sont les suivants : corps allongé à . oyau situé plus près de l'extrémité antérieure que de l'extrémité postérieure ; noyau occupant toute la largeur du corps protoplasmatique ; blépharoplaste situé à une très petite distance de l'extrémité postérieure et toujours accolé à la paroi ; flagelle libre assez long. Toujours monomorphe.

A l'examen à l'état frais, le trypanosome ne présente que peu de mouvements de torsion sur lui-même, que l'on peut comparer aux mouvements du $T$. congolense. Très pathogène pour le porc (une forte épidémie a éclaté pendant mon absence), l'inoculation de $5 \mathrm{cc}$. de sang très infecté au cobaye et au lapin est restée sans résultat. L'infection étant actuellement disparue, je n'ai pu poursuivre l'étude de son action pathogène sur d'autres animaux de laboratoire.

Laboratoire de Bactériologie d'Eiisabelhville (Congo Belge). 